\title{
gacerm \\ Colonic stenting no better than emergency surgery?
}

The high incidence of colorectal cancer and the high number of patients presenting with a bowel obstruction (7-29\% of patients with colorectal cancer) requires the development of novel techniques to improve the quality of life of these patients. One such technique is the use of colonic stenting as a bridge to elective surgery, to avoid the necessity of emergency surgery in eligible patients.

The use of colonic stenting might not be viewed as being very novel, as it has been in use since the early 1990s. In uncontrolled studies in patients with malignant obstruction of the left side of the colon, the use of a stent prior to elective surgery decreased mortality, morbidity and the number of colostomies. However, this technique had not been examined in a prospective randomized trial, which prompted a team led by Jeanin van Hooft to design a trial of this type. As van Hooft describes, "we realized that the evidence for colonic stenting as a bridge to surgery was frail, no randomized controlled trials to compare this new
4 Perhaps surprisingly ... none of the end points was significantly different... 77

technique with the gold standard seem to have been conducted."

Patients at 25 Dutch cancer centers with an acute obstructive left-sided lesion suspected to be malignant were recruited onto the trial. Owing to the nature of the treatment, neither the patient nor the surgeon could be blinded to the therapy; however, the randomization (1:1 to stenting or emergency surgery) was conducted centrally by the principal investigator using the internet.

The primary outcome of this trial was quality of life as assessed using a European Organisation for Research and Treatment of Cancer questionnaire. Other end points were mortality, morbidity, and stoma rate. Perhaps surprisingly, when considering previous reports, none of the end points was significantly different between the two groups. However, van Hooft states, "these findings are consistent with the only other recently published randomized controlled trial in this setting."

The trial was terminated early owing to a concern from the data safety monitoring committee that the colonic stenting cohort had a higher morbidity than the emergency surgery group. This finding was not observed with longer follow up, but van Hooft notes that this trial "raises concerns regarding overt and silent perforations in the colonic stenting group."

The results were summarized by van Hooft as: "in our opinion, colonic stenting can be used as an alternative to emergency surgery, but it should be used with caution." Further work should assess the treatment in specific patient groups.

Rebecca Kirk

Original article van Hooft, J. E. et al. Colonic stenting versus emergency surgery for acute left-sided malignant colonic obstruction: a multicentre randomised trial. Lancet Oncol. 12, 344-352 (2011) 